\title{
Review of the Dental Trauma Guide; an interactive guide to evidence-based trauma management
}

\author{
Peter F Daya \\ Sophy K Barberb \\ ${ }^{a}$ Department of Paediatric Dentistry, Leeds Dental Institute and Bradford District Care Trust Salaried Dental Service, UK \\ ${ }^{b}$ Department of Orthodontics, Leeds Dental Institute and Pinderfields Hospital, Wakefield Dental Service, UK
}

The complexities of dental trauma Traumatic dental injuries are common, with between $6-34 \%$ of children in the UK aged 8-15 experiencing damage to their permanent teeth..$^{1-3}$ Over three quarters of all traumatic oral injuries occur in childhood. ${ }^{4}$

For the clinician providing the initial emergency care the experience can be extremely stressful as often the patient is being seen as an emergency between other waiting patients and is not familiar to the clinician. The patient will be upset and in pain, and paediatric patients may be inexperienced with dental treatment and anxious. Additionally parents are frequently upset or even absent if the accident occurred away from home. Furthermore, traumatic dental injuries represent a complex diagnostic problem with a multitude of treatment options to choose from. For example, there are nine different kinds of fractures and six luxation injuries which can combine in 54 different ways; each resulting injury has specific treatment demands and prognoses with respect to pulp and periodontal healing. The management can be further complicated by trauma to multiple teeth, with different injuries, requiring treatment prioritisation. It is important to get this right, because providing appropriate initial emergency care significantly improves prognosis. ${ }^{5}$

Dental trauma almost always involves the upper anterior teeth ${ }^{6}$ and therefore can have a considerable effect on dental and facial aesthetics. This frequently coincides with adolescence and can have a significant impact for some children on their quality of life. ${ }^{7}$ Furthermore there are financial implications to health services and families in providing acute and follow-up care for such injuries. ${ }^{8,9}$ Finally there is amongst general practitioners the reported expectation that acute care for traumatic dental injuries lies within their remit and skill mix. ${ }^{10}$

It is therefore difficult for clinicians to keep fully updated on the 'evidence based'

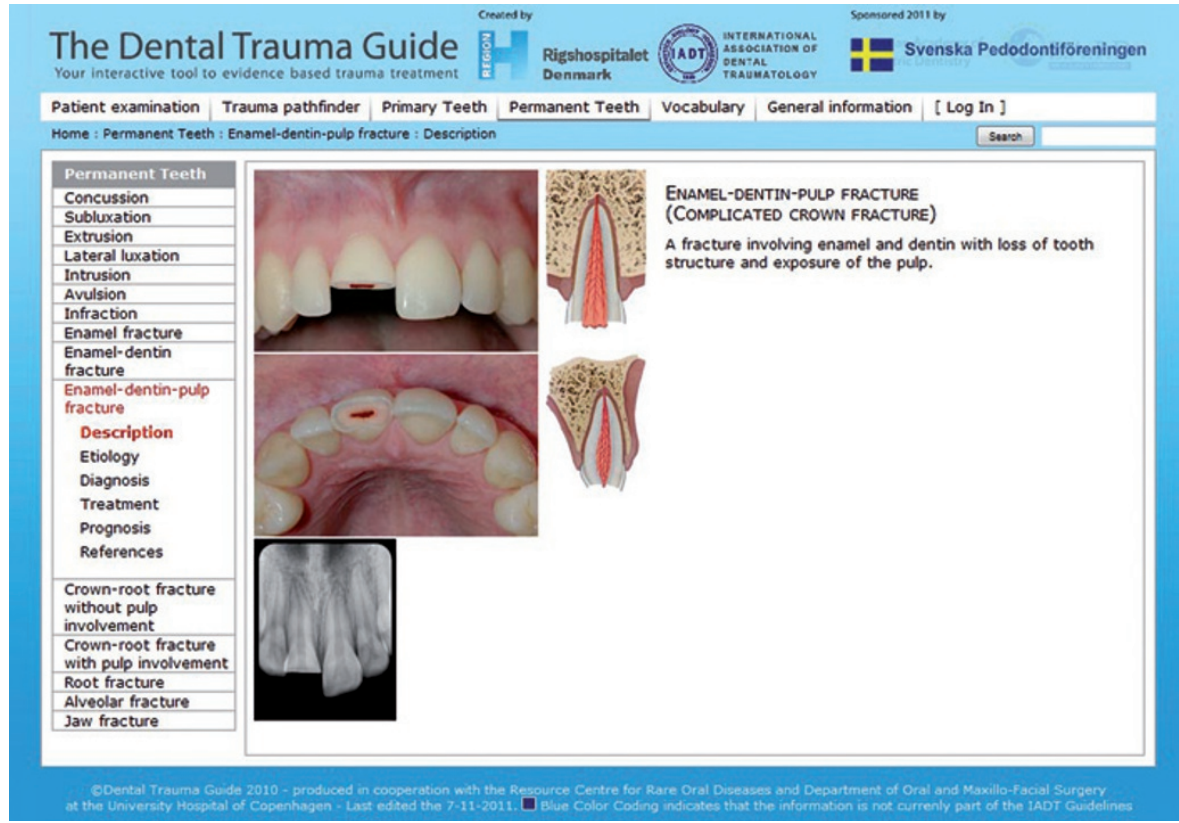

Figure 1 The layout of the website with the different injuries listed down the left hand side. When one of these injuries is tapped, a drop-down list detailing the description, etiology, diagnosis, treatment, prognosis and references are listed. This webpage provides a description of an enamel dentine pulp injury: http://www.dentaltraumaguide.org/Permanent_enameldentin-pulp_fracture_Description.aspx

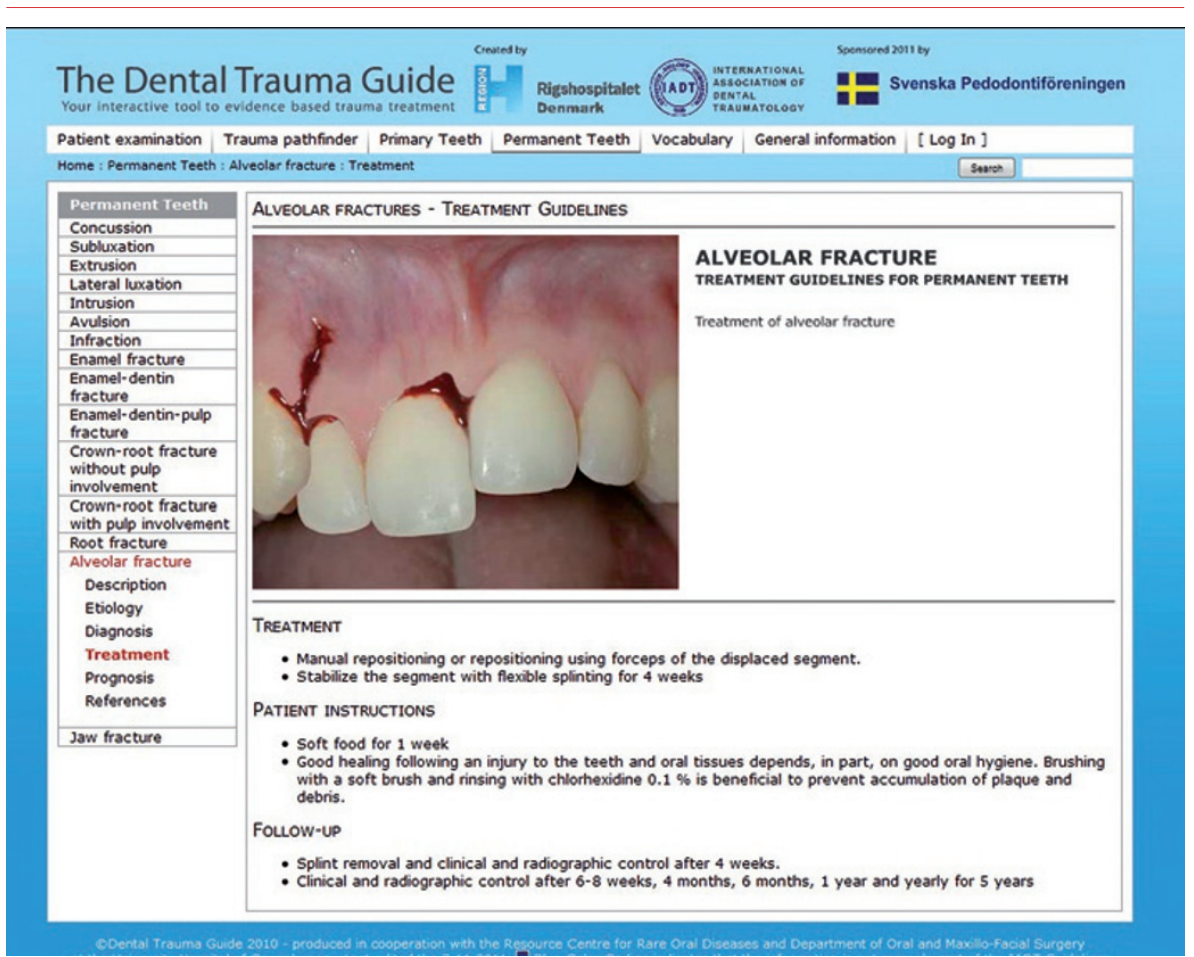

Figure 2 An animated description of how to treat / reposition an alveolar fracture. http://www.dentaltraumaguide.org/Permanent_Alveolar_fracture_Treatment.aspx 
approach to diagnosis and treatment, and feel confident managing dental trauma.

Dental Trauma Guide (www.dentaltraumaguide.org)

For the reasons discussed above, the unpredictable nature of dental trauma and the rarity that significant injuries will present in general practice, this website is an excellent resource. It is easy to use and guides the clinician through the examination, making the correct diagnosis and identifying the appropriate treatment. It is free to access on the internet and is simple to use. We highly recommend that all dentists visit the website and save it as one of their favourites on their work computer. This article will discuss five areas which we feel are some of the best features of the website together with discussing the scientific basis upon which it has been developed.

\section{Ease of use}

The website (www.dentaltraumaguide.org) is easy to find through most search engines by simply entering 'dental trauma'. The site is simple and straightforward to use with logical steps connecting the various sections. Clear section headings (Patient Examination, Trauma Pathfinder, Primary Teeth, Permanent Teeth, Vocabulary and General Information) exist on tabs along the top which can be clicked to access the required information. For both the primary and permanent dentitions a vertical index down the left side provides shortcut access to the various dental injuries, which are then further subdivided into: Description; Aetiology; Diagnosis; Management; and Prognosis (Figure 1). The illustrations and animations are clear and are supported by simple text information (Figure 2). Sufficient information is provided without overwhelming the user with evidence whilst references have been made available at the end of each section.

\section{Examination}

Diagnosis is essential before the appropriate management can be provided. The examination section provides step-by-step guidance through a systematic examination of the patient, to ensure that all information is gathered and to prevent omissions. Important questions to ask regarding the circumstances of the injury are provided with an indication of any significant answers. A

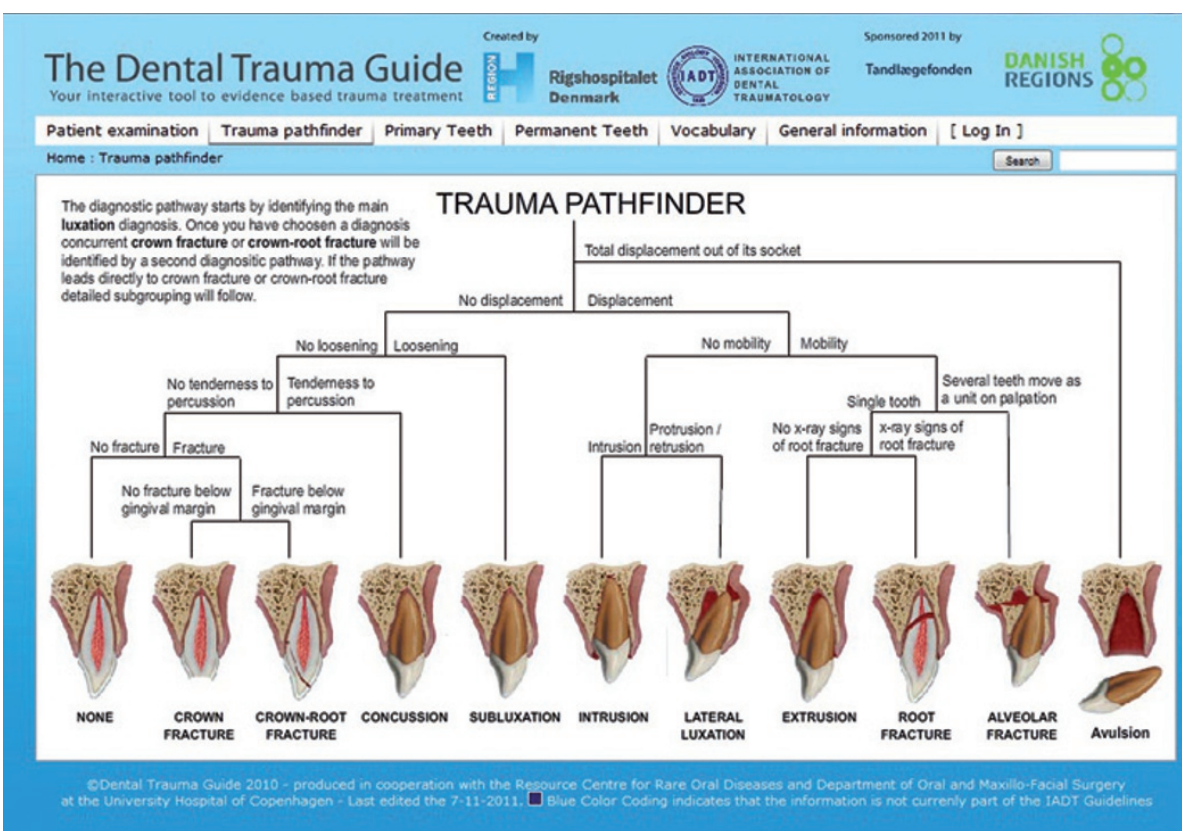

Figure 3 The trauma pathfinder which guides clinicians to identify the correct periodontal diagnosis for each injured tooth. On tapping on each of the diagnosis a further flow charted is opened which helps to identify any hard tissue injury where a periodontal injury has already been identified. http://www.dentaltraumaguide.org/Trauma_Pathfinder.aspx

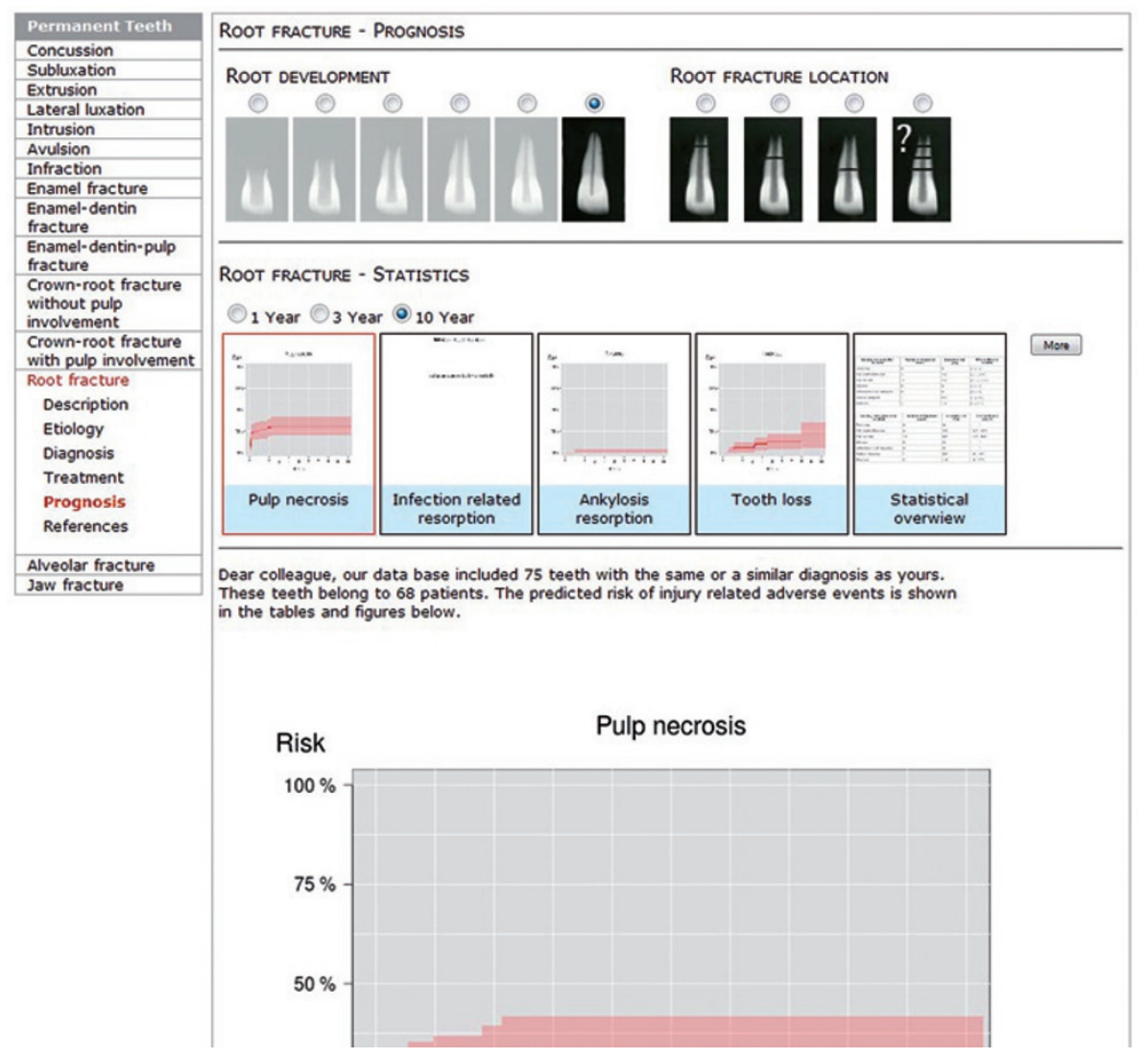

Figure 4 The prognosis for a root fracture tooth. The clinician must identify the root maturity and the location of the fracture. With this information the website will identify how many teeth from how many patients the outcome data is based upon. Furthermore details of the number of patients at each time point $(1,3,10$ years) are available. The estimation of pulp survival, periodontal healing and tooth survival is provided with a confidence interval to help clinicians to identify the strength of this estimation. http://www.dentaltraumaguide.org/Permanent_Root_fracture_ Prognosis.aspx

standardised clinical and radiological examination is described. By working through these stages, the clinician will have gathered sufficient information to make a sensible attempt at diagnosis. Where clinicians need further help with identifying the diagnosis 


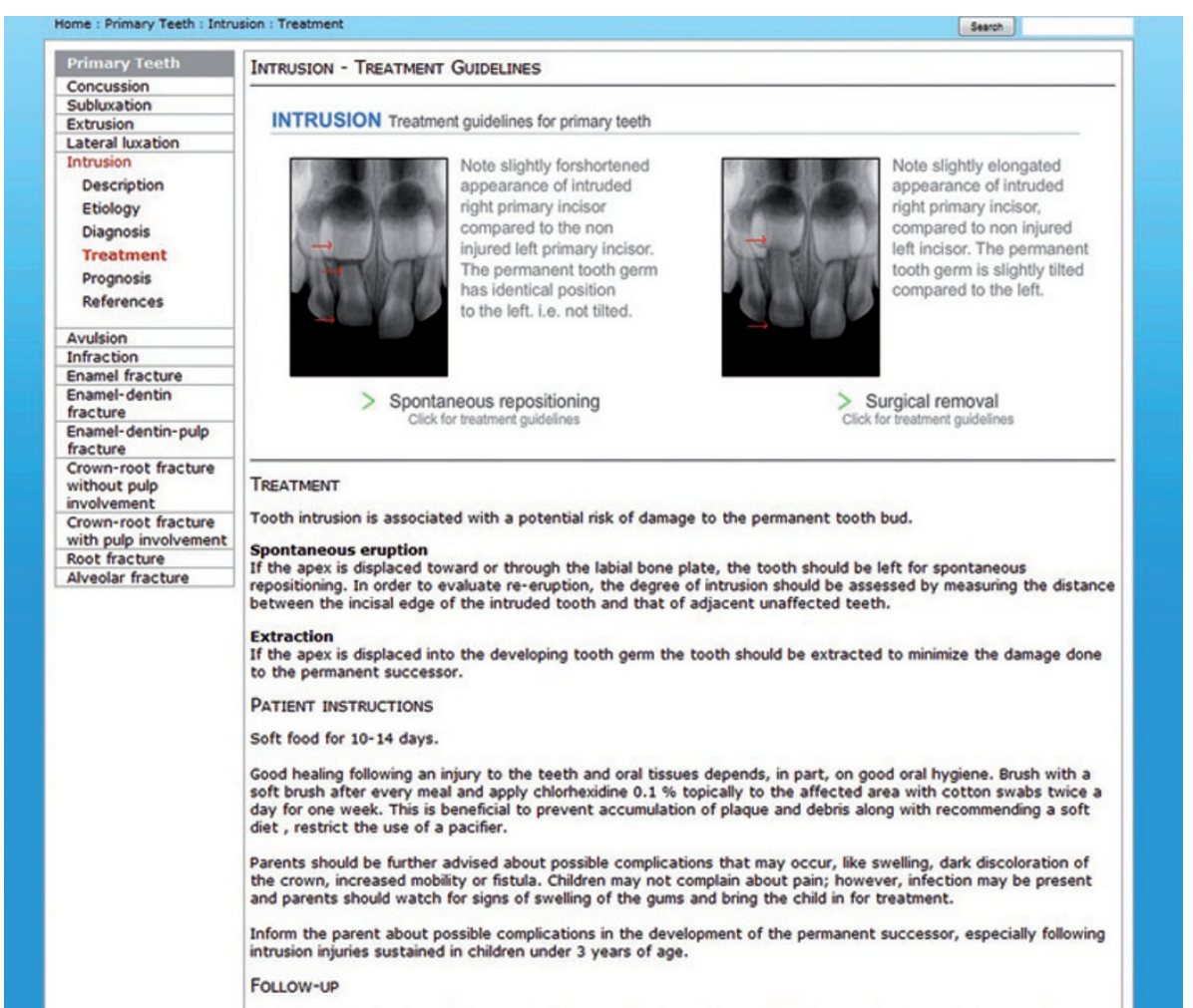

Figure 5 How clinicians can identify the direction of intrusion for the intruded primary tooth, and whether the permanent tooth is likely to have been directly involved. This difficult clinical decision strongly influences treatment to be provided; whether the child has an extraction carried out or the tooth is left to spontaneously erupt. http://www.dentaltraumaguide.org/ Primary_Intrusion_Treatment.aspx

the trauma pathfinder, discussed in the next section, is a valuable resource.

\section{Trauma pathfinder}

The trauma pathfinder guides clinicians, through a series of 'clickable' steps, to the diagnosis for both periodontal and hard tissue injuries which can occur independently or together within the same tooth. A flowchart with questions and illustrations directs the clinician to the correct diagnosis (see Figure 3). First a luxation injury can be identified by determining tooth displacement and mobility. Once this is determined, by clicking on this diagnosis, a second diagnostic pathway appears giving further subgroup options on the presence of various fractures in the crown/root. This pathway allows complex injuries to be systematically built-up so all aspects of the injury are identified and classified. Once a diagnosis has been reached, guidelines for treatment can be followed with animated illustrations and simple instructions. If there are different management strategies for the same injury that depend on other factors, for example avulsion with a closed apex rather than open apex, the specific guidance for that criterion can be followed. Information regarding treatment objectives, patient instructions and follow-up are given.

\section{Prognosis}

Presenting information on the prognoses for different traumatic injuries provides an excellent resource for all clinicians who treat dental trauma (Figure 4). Once the diagnosis has been identified, the website requires the clinician to record other prognostic information, for example, stage of root development. With this information, prognostic outcomes including periodontal and pulpal healing and tooth survival are generated for the exact injury the patient has suffered. This is given in graph and table format with both a point estimate and a confidence interval. These risk estimates have been derived from survival statistics of different trauma type sub-groups, taken from a database with 4,000 patients' long-term trauma outcome data.

The information on prognosis for different types of injury supports clinicians to have informed discussions with parents and patients about likely outcomes, expected future treatment and associated financial and indirect costs. It is also useful for supporting the interpretation of clinical and radiographic findings for patients attending review appointments.

\section{Vocabulary}

There are a number of nomenclature systems used to describe dental injuries which can cause confusion for those unfamiliar with trauma. In addition, accurate terminology aids communication between clinicians regarding the diagnosis, prognosis and treatment provided which can be essential for the long-term management and follow-up of patients with dental trauma. The vocabulary section aims to help practitioners by:

- Classifying fracture, luxation and soft tissue injuries with a description of each injury

- Describing the possible sequelae of dental trauma

- Defining common terms used in relation to tests, treatment, healing and tooth development disturbances.

Practitioners can access this to enable accurate referrals and to aid communication with patients.

\section{Primary teeth}

Similar to the pathfinder for permanent teeth, there is a description of the injuries, with an outline of aetiology, diagnosis and management of primary teeth. High quality pictures of radiographs illustrate the importance of determining the position of the primary tooth in relation to the permanent successor when choosing the appropriate treatment options (Figure 5). Information on prognosis of primary teeth injuries is currently under construction and should be available soon.

\section{Limitations of the Dental Trauma Guide}

The Dental Trauma Guide is a considerable resource to aid diagnosis and management of trauma, but as with all tools, it is only designed to provide assistance to a sound basis of knowledge. The guide is unable to provide sufficient combinations of traumatic injury to cover all the potential situations which may arise, and currently there is no tool to assist with prioritising multiple injuries. This is unsurprising as treatment planning is influenced by the availability of clinical resources as well as the multitude of combination injuries possible. 
The evidence base to support many treatment interventions in dental trauma is limited and is frequently based on animal or clinical case series. The Dental Trauma Guide website, in the vast majority of cases, follows the International Association of Dental Traumatology (IADT) guidelines on management. The website shows animated descriptions of treatment protocols. Whilst the references are provided, it is unfortunate that they are all provided for each injury at the end of the sections and so it is not clear how the evidence and recommendations are linked.

The prognostic data provide a rich and excellent resource which will help clinicians and patient / parents alike. The use of confidence intervals allows clinicians to visualise the strength of the evidence for each potential outcome. Furthermore, for each combination of injuries the website records how many teeth and patients with a similar history the prognostic data are based upon at each of the time points. As with many clinically relevant outcomes, long term outcomes can only be derived where cases have attended regular follow-ups. Consequently it is unknown how similar injuries fared in patients who failed to return for followup care or are not included in the prognosis part of the website. As described in the development of the website, the prognostic data are based on only $10 \%$ of patients who attend the Copenhagen centre over the clinical lifetime of Dr. Andreasen. Unfortunately the data used to generate the prognostic out- comes for some injures are based on unpublished papers. Consequently these have not been peer reviewed nor are they available for further reading and research. The reference lists mainly concentrate on the published work of the author although other important papers are included. Frustratingly some papers are 'in preparation' and therefore unavailable to the reader.

The treatment advice in most cases follows IADT guidelines and for situations where different approaches are available a table of advantages and disadvantages of each is provided. This is very helpful to readers. In one or two places a gremlin still exists with the trauma guide but these are remarkably few. Despite treatment guidelines identifying the depth of intrusion as an important variable in determining what treatment to provide, the prognostic outcome data do not ask clinicians to record this information when calculating the likely outcome. This disparity is not easy to explain.

The website still advocates three to four radiographs being taken for each traumatic injury as a standard baseline for each acute trauma presentation. This is probably excessive and unrealistic. Furthermore the diagnostic yield after two radiographs per tooth is low and the further information in the vast majority of cases will not change the treatment provided.

\section{Conclusion}

In summary the dental trauma guide is a tremendous new resource for all clinicians who treat dental trauma. We can only congratulate the authors for making such a valuable resource so easily available to clinicians. We hope that with this support, clinicians will feel more confident to provide acute trauma management and identify those injuries or patients where specialist help would be beneficial.

1. O'Brien, M. Children's Dental Health in the United Kingdom. London: HMSO 1993.

2. Hamilton FA, Hill FJ, Holloway PJ. An investigation of dento-alveolar trauma and its treatment in an adolescent population. Part 1: the prevalence and incidence of injuries and the extent and adequacy of treatment received. Br Dent J 1997; 182: 91-95.

3. Chadwick BL, White DA, Morris AJ, Evans D, Pitts NB. Non-carious tooth conditions in children in the UK, 2003. Br Dent J 2006; 200: 379-384.

4. Petersson EE, Andersson L, Sorensen S. Oral injuries in relation to non oral injuries. Swed Dent / 1997; 21: 55-68.

5. Andreasen JO, Andreasen FM, Skeie A, HjortingHansen E, Schwartz O. Effects of treatment delay upon pulp and periodontal healing of traumatic dental injuries - a review article. Dent Traumatol 2002; 18: $116-128$

6. Jacobsen I, Andreasen JO. Traumatic injuries examination, diagnosis and immediate care. In Koch G, Poulsen S. (eds) Paediatric dentistry, a clinical approach. pp 351-380. Copenhagen: Munksgaard 2001.

7. Rodd HD, Barker C, Baker SR, Marshman Z, Robinson PG. Social judgements made by children in relation to visible incisor trauma. Dent Traumatol 2010; 26: 2-8.

8. Nguyen PM, Kenny DJ, Barrett EJ. Socio-economic burden of permanent incisor replantation on children and parents. Dent Traumatol 2004; 20: 123-133.

9. Wong FS, Kolokotsa K. The cost of treating children and adolescents with injuries to their permanent incisors at a dental hospital in the United Kingdom. Dent Traumatol 2004; 20: 327-333.

10. Jackson NG, Waterhouse PJ, Maguire A. Management of dental trauma in primary care: a postal survey of general dental practitioners. Br Dent / 2005; 198: 293-297.

Evidence-Based Dentistry (2011) 12, 117-120. doi:10.1038/sj.ebd.6400830. 